\title{
Association of spinal instability due to metastatic disease with increased mortality and a proposed clinical pathway for treatment
}

\author{
Patricia Zadnik Sullivan, MD, ${ }^{1}$ Ahmed Albayar, MD, ${ }^{1}$ Ashwin G. Ramayya, MD, PhD, ${ }^{1}$ \\ Brendan McShane, BS, ${ }^{2}$ Paul Marcotte, MD, ${ }^{1}$ Neil R. Malhotra, MD, ${ }^{1}$ Zarina S. Ali, MD, MS, ${ }^{2}$ \\ H. Isaac Chen, MD, ${ }^{1}$ M. Burhan Janjua, MD, ${ }^{3}$ Comron Saifi, MD, James Schuster, MD, PhD, ${ }^{1}$ \\ M. Sean Grady, MD, ${ }^{1}$ Joshua Jones, MD, ${ }^{5}$ and Ali K. Ozturk, MD²
}

\begin{abstract}
Departments of ${ }^{1}$ Neurosurgery and ${ }^{5}$ Radiation Oncology, University of Pennsylvania; Departments of ${ }^{2}$ Neurosurgery and ${ }^{3}$ Orthopedic Surgery, Pennsylvania Hospital, Philadelphia, Pennsylvania; and ${ }^{4}$ Department of Neurological Surgery, University of Texas Southwestern, Dallas, Texas
\end{abstract}

\begin{abstract}
OBJECTIVE Multidisciplinary treatment including medical oncology, radiation oncology, and surgical consultation is necessary to provide comprehensive therapy for patients with spinal metastases. The goal of this study was to review the use of radiation therapy and/or surgical intervention and their impact on patient outcomes.

METHODS In this retrospective series, the authors identified at their institution those patients with spinal metastases who had received radiation therapy alone or had undergone surgery with or without radiation therapy within a 6-year period. Data on patient age, chemotherapy, surgical procedure, radiation therapy, Karnofsky Performance Status (KPS), primary tumor pathology, Spinal Instability Neoplastic Score (SINS), and survival after treatment were collected from the patient electronic medical records. N - 1 chi-square testing was used for comparisons of proportions. The Student t-test was used for comparisons of means. A p value $<0.05$ was considered statistically significant. A survival analysis was completed using a multivariate Cox proportional hazards model.
\end{abstract}

RESULTS Two hundred thirty patients with spinal metastases were identified, 109 of whom had undergone surgery with or without radiation therapy. Among the 104 patients for whom the surgical details were reviewed, 34 (33\%) had a history of preoperative radiation to the surgical site but ultimately required surgical intervention. In this surgical group, a significantly increased frequency of death within 30 days was noted for the SINS unstable patients $(23.5 \%)$ as compared to that for the SINS stable patients $(2.3 \% ; p<0.001)$. The SINS was a significant predictor of time to death among surgical patients (HR 1.11, $p=0.037$ ). Preoperative KPS was not independently associated with decreased survival $(p>0.5)$ on univariate analysis. One hundred twenty-six patients met the criteria for inclusion in the radiation-only analysis. Ninetyeight of these patients $(78 \%)$ met the criteria for potential instability $(\mathrm{PI})$ at the time of treatment, according to the SINS system. Five patients $(5 \%)$ with $\mathrm{PI}$ in the radiation therapy group had a documented neurosurgical or orthopedic surgery consultation prior to radiation therapy.

CONCLUSIONS At the authors' institution, patients with gross mechanical instability per the SINS system had an increased rate of 30-day postoperative mortality, which remained significant when controlling for other factors. Surgical consultation for metastatic spine patients receiving radiation oncology consultation with $\mathrm{Pl}$ is low. The authors describe an institutional pathway to encourage multidisciplinary treatment from the initial encounter in the emergency department to expedite surgical evaluation and collaboration.

https://thejns.org/doi/abs/10.3171/2019.11.SPINE19775

KEYWORDS metastatic spine disease; Spine Instability Neoplastic Score; comprehensive; pathway; oncology

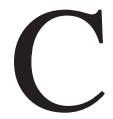
ANCER care in the modern era has become a multidisciplinary effort, as patients are surviving longer after primary diagnosis. ${ }^{4,20}$ Historically, surgery, chemotherapy, and radiation therapy have been utilized together to improve survival and quality of life for patients with metastatic spine disease. As new technology has become available, the decision-making process has become increasingly complex. Although overt mechanical instability or significant neurological impairment is a well-established indicator for surgical intervention, many patients

ABBREVIATIONS MFRT = multiple-fraction radiation therapy; OIS = Oncology Information System; PI = potential instability; SFRT = single-fraction radiation therapy; SINS = Spinal Instability Neoplastic Score; SRT = stereotactic radiotherapy.

SUBMITTED July 7, 2019. ACCEPTED November 20, 2019.

INCLUDE WHEN CITING Published online February 14, 2020; DOI: 10.3171/2019.11.SPINE19775. 
live for years with back pain and known metastatic disease in the bony spine. For these patients, it can be difficult to determine the ideal time for surgery.

In a landmark study demonstrating the efficacy of surgery for patients with spinal metastases, Patchell and colleagues showed that laminectomy with or without fusion plus concomitant radiation therapy leads to preserved ambulation and a decrease in narcotic and steroid use. ${ }^{21}$ Since publication of that study, multiple groups have tried to optimize the combination of radiation therapy and surgery using algorithms to incorporate complex patient factors. The NOMS framework from the Memorial Sloan Kettering Cancer Center takes into account neurological, oncological, mechanical, and systemic considerations in formulating an ideal treatment plan. ${ }^{17}$ Using the NOMS system, surgeons and radiation oncologists can recommend radiation, stereotactic radiosurgery, or surgery with or without stabilization. The Spinal Instability Neoplastic Score (SINS) is another system, developed by the Spine Oncology Study Group (Table 1). ${ }^{5}$ The SINS classification system is meant to provide guidance on the need for surgery in metastatic spine disease and contains six variables: lesion location, pain characterization, type of bone lesion (lytic vs sclerotic), radiographic spinal alignment, degree of vertebral body collapse, and posterior column involvement. Graded on a scale from 1 to 18 , the total SINS signifies stable (1-6), potentially unstable (7-12), and unstable (13-18) conditions. The SINS system has proven to be an effective tool for clinicians to assess spine instability and, importantly, has shown good inter- and intraobserver reliability. 2,6,8,22

Fundamentally, such scoring systems are meant to guide surgical decision-making and to control the switch from medical to surgical care. Historically, surgeons have entered the treatment team when acute neurological deterioration or frank instability is noted. Metastatic spinal cord compression can result from epidural growth of a tumor or the fracture and subluxation of diseased vertebral bodies due to lytic destruction of bone. Spinal cord compression occurs in up to $10 \%$ of cancer patients, resulting in various degrees of neurological compromise and pain. ${ }^{15,18}$ Stabilization using pedicle screws and rods may be required in patients with mechanical instability (fracture, subluxation, or kyphosis); however, surgery carries up-front risk and may not be offered to patients with a poor prognosis. Patient preferences, the current metastatic burden, and oncological medication regimens can further complicate the radiation versus surgery discussion. However, as radiation treatments continue to advance, the indications for aggressive resection will be redefined. Radiation therapy can be administered in single (SFRT) or multiple (MFRT) fractions or as stereotactic radiotherapy (SRT) to reduce some types of cancer-related pain, to treat local disease, and, with SRT, to obtain high rates of local tumor control. ${ }^{3}$

In the absence of neurological dysfunction or overt instability, radiation therapy remains the least invasive option in amenable tumors to achieve tumor control and pain reduction. Unlike surgery, radiation can also be used to treat multiple adjacent levels in multifocal disease. SRT, SFRT, and MFRT can be tailored to patient goals and
TABLE 1. SINS system

\begin{tabular}{ll}
\hline \multicolumn{1}{c}{ Element of Score } & Score \\
\hline Lesion location & \\
Junctional (occiput-C2, C7-T2, T11-L1, L5-S1) & 3 \\
Mobile spine (C3-C6, L2-L4) & 2 \\
Semi-rigid (T3-T10) & 1 \\
Rigid (S2-S5) & 0 \\
\hline Pain relief w/ recumbency \&/or pain w/ movement/loading & \\
of the spine & 3 \\
Yes & 1 \\
$\quad$ No (occasional pain but not mechanical) & 0 \\
$\quad$ Pain-free lesion & \\
\hline Bone lesion quality & 2 \\
Lytic & 1 \\
Mixed (lytic/blastic) & 0 \\
Blastic & \\
\hline Radiographic spinal alignment & 4 \\
Subluxation/translation present & 2 \\
De novo deformity (kyphosis/scoliosis) & 0 \\
Normal alignment & \\
\hline Vertebral body collapse & 3 \\
$>50 \%$ collapse & 2 \\
<50\% collapse & \\
No collapse w/ $>50 \%$ body involved & 1 \\
None of the above & 0 \\
\hline Posterolateral involvement of spinal elements & \\
Bilateral & \\
Unilateral & \\
None of the above & \\
\hline
\end{tabular}

Reprinted with permission from Fisher CG, DiPaola CP, Ryken TC, et al: A novel classification system for spinal instability in neoplastic disease: an evidence-based approach and expert consensus from the Spine Oncology Study Group. Spine 35 (22): E1221-E1229. https://journals.Iww.com/ spinejournal/pages/default.aspx.

prognoses, although there is significant variability among radiation treatment options in terms of adverse effects and outcomes. ${ }^{3,9}$ Radiation oncologists, like surgeons, often face problematic discussions regarding prognosis and goals with patients with metastatic spine disease and may recommend a palliative course or a single fraction if the patient is near the end of life. Pure bone pain from diseased vertebral bodies is likely to improve significantly with both SFRT and MFRT. ${ }^{13}$ In addition, palliative radiotherapy alone is likely to stabilize or improve neurological deficits for a large percentage of patients with spinal cord compression. In the SCORE2 (Study of Comparative Treatments for Retinal Vein Occlusion 2) and SCORAD III (A randomised phase III trial of single fraction radiotherapy compared to multifraction radiotherapy in patients with metastatic spinal cord compression) randomized trials, both SFRT and MFRT improved ambulatory status at 8 weeks in patients with metastatic spine disease. ${ }^{12,21}$

In the present study, we examined care patterns for patients with metastatic spine disease at multiple hospitals within a single academic institution. Patients who had undergone radiation treatment and/or surgery were reviewed in a retrospective fashion. Furthermore, as a surrogate for 
biomechanical stability at the time of radiation or before surgical intervention, SINSs were calculated retrospectively. We identified patients with poor outcomes after surgery and used these data to identify areas of improvement for patients with metastatic cancer to the spine and to create pathways to improve and streamline their care at our institution.

\section{Methods}

All patient data were acquired in accordance with the University of Pennsylvania Institutional Review Board. Patients from three hospitals within our institution were included: Hospital of the University of Pennsylvania, Pennsylvania Hospital, and Penn Presbyterian Medical Center. Using PennSeek, we identified patients meeting the search criterion for "metastatic spine tumor" with a documented radiation oncology encounter between January 2010 and January 2017. Patients who had undergone surgery for decompression with or without stabilization from January 2011 to December 2016 and meeting the search criterion for "metastatic spine tumor" were also identified using PennSeek. The "index site" was defined as the spinal region inclusive of the radiation treatment field (i.e., cervical, thoracic, lumbar). Patient medical records and pretreatment radiographs were reviewed to determine if surgical decompression or stabilization had been performed at the index site. Patients were excluded from the radiation-only treatment group if there was evidence of prior laminectomy or stabilization at the index site. Details of the surgical procedure were reviewed for all patients who had undergone surgery for spinal metastatic disease and met the criteria for inclusion in the SINS analysis.

The SINS was calculated as previously described..$^{2}$ The scoring system is presented in detail in Table 1. The pain component of the SINS was calculated from the patient's electronic medical record. Specifically, if patients reported pain with ambulation or movement, the pain score was marked as "yes" for mechanical pain. If the patient was on short- or long-acting narcotic medication or described pain at rest and did not report pain with ambulation or movement, the pain score was marked as "no." If the patient did not complain of pain and was not on any short- or long-acting narcotics, the pain score was marked as "painfree lesion."

Radiation treatment plans and beam contours at the index site were identified from the ARIA Oncology Information System (ARIA OIS; Varian Medical Systems). Dates of service were matched to the radiation treatment plan, and CT simulation scans were obtained in ARIA OIS. These scans were utilized to identify lesion location, lesion quality, alignment, vertebral body collapse, and posterior element involvement to assign an SINS. Note that an SINS $>6$ was accepted as indicating potential instability (PI). Of note, the Bilsky score was not calculated for the degree of epidural spinal cord compression, as MRI is not routinely archived for patients in the radiation oncology system at our institution.

Evidence of neurosurgical or orthopedic consultation from the patients' electronic medical records was reviewed. All hospitals within our institution utilize the same electronic medical record, and clinic notes, emergency department visits, outpatient phone calls, and all inpatient visits are recorded in the electronic medical record. If an inpatient consultation was recorded for neurosurgical or orthopedic surgical evaluation at the index site, the patient was scored as having a surgical consultation. Outpatient and inpatient radiation oncology notes were reviewed and searched for the terms "neurosurgery," "surgeon," "orthopedic," and "consult." Any notes including these terms were reviewed to confirm consultation for the index site (as defined above). Informal consultations, such as phone calls to review films or undocumented discussion at a tumor board, were not captured in the electronic record.

$\mathrm{N}-1$ chi-square testing was used for comparisons of proportions. The Student t-test was used for comparisons of means. A p value $<0.05$ was considered statistically significant. Survival analysis using a multivariate Cox proportional hazards model was performed to assess whether the SINS was a significant predictor of time to death even when accounting for other clinical factors. To identify clinical factors to include in the multivariate analysis, several univariate Cox proportional hazards regressions were performed to identify variables that were predictors of time to death $(\mathrm{p}<0.15)$.

\section{Results}

\section{Radiation-Only Group}

We sought to determine how many patients undergoing radiation therapy exhibited PI according to the SINS criteria. One hundred seventy-nine patients with metastatic spine tumors were identified in the radiation-only treatment group. One hundred twenty-six patient electronic medical records contained CT imaging within the ARIA OIS system for scoring according to the SINS system. The most common pathology was lung cancer (41 [32.5\%]), followed by "other" (29 [23\%]), breast (27 [21.4\%]), and prostate cancer (18 [14.3\%]; Table 2). The population was $51 \%$ female (64), and the average age was 65.5 years (range 27.9-93.3 years). At the time of treatment, 2 patients (1.6\%) were unstable (SINS 13-16), 98 patients (77.8\%) met criteria for PI (SINS 7-12), and 26 patients $(20.6 \%)$ were stable (SINS $\leq 6)$. The distribution of SINSs is listed in Table 2.

Surgical intervention is typically offered to patients with more than 3-6 months of expected survival; thus, we examined how many patients with PI had documented survival more than 6 months after radiation treatment. Among the 98 radiation-only patients with metastatic spine disease and PI, the mean age at treatment was 65.9 years (range 27.9-92.3 years) and mean survival after radiation treatment was 15 months (range 5 days-102 months; Table $3)$. Eighty-five patients (87\%) in the PI group reported mechanical back pain at the time of treatment. Among the 98 patients with PI, only $5(5 \%)$ had a documented neurosurgical or orthopedic surgery consultation for spinal disease prior to radiation therapy. Two patients $(2 \%)$ were referred for neurosurgical consultation after radiation therapy.

Forty-five PI patients survived more than 6 months after radiation, with an average survival of 29.7 months 
TABLE 2. Summary of characteristics in patients treated for spinal metastases

\begin{tabular}{|c|c|c|c|c|c|}
\hline \multirow[b]{2}{*}{ Variable } & \multicolumn{2}{|c|}{ Surgery } & \multicolumn{2}{|c|}{ Radiation } & \multirow{2}{*}{$\begin{array}{c}p \\
\text { Value }^{*}\end{array}$} \\
\hline & No. & $\%$ & No. & $\%$ & \\
\hline Total no. of patients & 109 & & 126 & & \\
\hline \multicolumn{6}{|l|}{ Pathology } \\
\hline Breast & 20 & 19.2 & 27 & 21.4 & 0.55 \\
\hline Colon & 4 & 3.8 & 6 & 4.8 & 0.64 \\
\hline Lung & 9 & 8.7 & 41 & 32.5 & $<0.0001$ \\
\hline Prostate & 8 & 7.7 & 18 & 14.3 & 0.089 \\
\hline Renal & 15 & 14.4 & 5 & 4.0 & 0.0075 \\
\hline Other & 48 & 46.2 & 29 & 23.0 & 0.0006 \\
\hline \multicolumn{6}{|l|}{ SINS } \\
\hline 1-6, stable & 7 & 6.7 & 26 & 20.6 & 0.003 \\
\hline $7-12, \mathrm{PI}$ & 63 & 60.6 & 98 & 77.8 & 0.0047 \\
\hline 13-16, unstable & 34 & 32.7 & 2 & 1.6 & $<0.0001$ \\
\hline
\end{tabular}

The surgical cohort was reduced to 104 patients because of unavailability of primary imaging for patient scoring.

* The $\mathrm{p}$ value reflects the comparison of proportions via the $\mathrm{N}-1$ chi-square test. A threshold of $p<0.05$ was considered statistically significant. Boldface type indicates statistical significance.

posttreatment (range 6.1-102.2 months). Only 1 (2\%) of these patients was seen for operative consultation by neurosurgery or orthopedic surgery before initiating radiation therapy.

\section{Surgery Cohort}

One hundred nine patients underwent surgery for metastatic spine tumors in the study period; 52 (48\%) of them had postoperative radiation therapy. Among the 104 patients for whom complete medical records were obtained, the most common pathology after "other" (46.2\%) was breast cancer (19.2\%), followed by renal (14.4\%) and lung (8.7\%; Table 2) cancer. Prostate $(7.7 \%)$ and colon (3.8\%) cancer were less common primary tumors. The mean age of the surgical patients was 60.8 years (range 31.6-87.4 years). One hundred four surgical patients met the criteria for inclusion in the SINS component of the study. Two patients underwent laminectomy alone for decompression,
2 patients underwent the anterior approach without posterior fusion, and 1 patient underwent focal decompression of the vertebral body via a posterior approach without posterior fusion. Ninety-nine patients underwent posterior fusion, and 54 of these patients underwent partial or complete corpectomy with posterior fusion. Among these patients, $34(33 \%)$ had a history of preoperative radiation to the surgical site but ultimately required surgical intervention. Seven patients $(6.7 \%)$ had an SINS indicating stability, whereas 63 patients $(60.6 \%)$ met the criteria for PI and 34 patients $(32.7 \%)$ met the criteria for instability.

Within the surgical cohort, 70 were classified as SINS stable (i.e., stable or PI, SINS < 13) and 34 patients were classified as SINS unstable (SINS $\geq 13$ ). Patient age and sex were not significantly different between these two groups (mean age 61 years, range $38-87$ years, $55 \%$ male and mean age 60 years, range 38-86 years, $44 \%$ male, respectively; all $\mathrm{p}>0.2$, Student t-test and chi-square test). Among the surgical patients, 8 required reoperation within 30 days for perioperative hematoma or infection (SINS unstable: 3 [9\%], SINS stable: 5 [7\%]). Fifty-five (52.9\%) of the surgical patients died: $46 \%$ of the SINS stable patients died during the follow-up period, with a mean time from surgery to death of 21 months (range 14 days -69 months), whereas $68 \%$ of the SINS unstable patients died, with a mean time from surgery to death of 15 months (range 2 days-68 months). We observed an increased frequency of in-hospital death within 30 days for SINS unstable patients $(23.5 \%)$ as compared to that for the SINS stable patients $(2.3 \% ; \mathrm{p}<0.001$, chi-square test).

We performed survival analysis using a multivariate Cox proportional hazards model to determine whether the SINS was a significant predictor of time to death even when accounting for other clinical factors. We did not find a significant relation between the following variables and time to death on univariate analysis $(p>0.2)$ : corpectomy, anterior approach, number of levels treated, location of tumor, local radiation after surgery, chemotherapy before surgery, chemotherapy after surgery, lung metastasis, brain metastasis, and other primary cancers (gastrointestinal, breast, liver, lymphoma, prostate, and thyroid cancer and small cell carcinoma). The relationship between preoperative Karnofsky Performance Status (KPS) and survival $(p>0.5)$ did not meet the criteria for inclusion in the multivariate regression analysis. The following variables

TABLE 3. Survival among patients treated for spinal metastases

\begin{tabular}{lccc}
\hline \multicolumn{1}{c}{ SINS } & $\begin{array}{c}\text { No. of } \\
\text { Patients (\%) }\end{array}$ & $\begin{array}{c}\text { Mean Age at Treatment } \\
\text { in Yrs (range) }\end{array}$ & $\begin{array}{c}\text { Mean Survival After Treatment } \\
\text { in Mos (range in days-mos) }\end{array}$ \\
\hline Radiation alone & & & NA \\
\hline Unstable lesion (SINS $\geq 13)$ & $2(2)$ & $71.1(60-83)$ & $15(5-102)$ \\
\hline PI (SINS 7-12) & $98(78)$ & $66(28-92)$ & NA \\
\hline Stable lesion (SINS $\leq 6)$ & $26(21)$ & $64(34-94)$ & $15(2-68)$ \\
\hline Surgery & & & $21(14-69)$ \\
\hline Unstable lesion (SINS $\geq 13)$ & $34(33)$ & $60(38-86)$ & \\
\hline Stable: stable or PI (SINS <13) & $70(67)$ & $61(38-87)$ & \\
\hline NA = not applicable. & &
\end{tabular}


were predictors of time to death on univariate analysis ( $\mathrm{p}$ $<0.15$ ) and were included in the multivariate analysis: local radiation before surgery (HR 2.1, $\mathrm{p}=0.008$ ), presence of liver metastasis (HR 1.9, $\mathrm{p}=0.03$ ), posterior instrumentation (HR 0.38, p = 0.07), age (HR 0.98, p = 0.11), male sex (HR 1.5, $\mathrm{p}=0.12)$, primary renal cancer (HR 0.46, $\mathrm{p}$ $=0.10)$, primary melanoma $(\mathrm{HR} 3.7, \mathrm{p}=0.01)$, primary lung cancer (HR 2.2, $\mathrm{p}=0.03$ ), and primary genitourinary cancer (HR 3.2, $\mathrm{p}=0.05$ ). The SINS was a significant predictor of time to death on multivariate analysis (HR 1.11, $\mathrm{p}=0.037$ ), as was primary lung cancer (HR 2.3052, $\mathrm{p}=$ $0.04)$. The following variables were nonsignificant: primary melanoma (HR $2.85, \mathrm{p}=0.06)$, primary genitourinary cancer (HR 3.0, p = 0.09), male sex $($ HR 1.69, $\mathrm{p}=0.07)$, and posterior instrumentation (HR 0.32, $\mathrm{p}=0.07$ ). The remaining variables were not significant predictors of time to death either $(\mathrm{p}>0.15)$ : presence of liver metastasis, age, and primary renal cancer.

\section{Comparison}

Patients in the radiation-only cohort were significantly older than the surgical patients (65.5 vs 60.8 years, $\mathrm{p}=$ $0.0055,95 \%$ CI $1.39-8.00$ ) There were significant differences in primary pathologies between surgical and radiation-only cohorts for lung, renal, and other pathologies (Table 2). The frequency of breast, colon, and prostate cancer was comparable. The rate of SINS unstable cases was significantly higher in the surgery cohort than in the radiation-only group (32.7\% vs $1.6 \%$; $\mathrm{p}<0.0001$; Table 2$)$.

\section{Discussion}

Surgical treatment for metastatic spine tumors has seen great improvement over the past several decades with highly effective instrumentation techniques such as pedicle screw placement, interbody cage placement, percutaneous screw insertion, and vertebroplasty. ${ }^{7,11,14}$ Yet these surgeries remain highly invasive in a population with limited life expectancy and complex medical comorbidities. Use of the SINS system to identify patients with instability - that is, surgical candidates - has proven to be a useful framework. Our data show that waiting for surgical intervention until patients exhibit gross instability is associated with a significantly higher rate of 30-day postoperative mortality. In addition to this finding, the rate of pre-radiation surgical consultation remains extremely low, even in patients with more than 6 months of life expectancy and distinct mechanical back pain.

These findings are not meant to disparage radiation treatment; rather, they highlight the need for increased collaboration between radiation oncologists and surgeons in treating the metastatic spine disease population. Radiation therapy is highly effective and can improve pain, maintain or improve ambulatory function, and treat the underlying disease process. ${ }^{15,18}$ In patients with multifocal disease, radiation can address multiple levels in a single therapy plan. ${ }^{1}$ However, radiation does have its limits. MFRT, while effective, can mean 10-15 days of continuous treatment, which is a significant burden for patients facing the end of life. For patients with prior radiotherapy, especially to the lungs or chest wall, conventional radiation treatment may be limited if the spinal cord has been exposed to a biologically effective dose exceeding 45-50 Gy during treatment of the primary cancer. Conventional radiation therapy is also limited in radio-resistant pathologies and in cases with a high degree of spinal cord compression. For SRT, separation surgery may be required to generate a safe distance between the spinal cord and the affected vertebral body. ${ }^{17,19}$ Delayed wound healing and postoperative infections following long-segment fusions in the spine are often feared complications, and the optimal timing of radiation therapy is essential to minimize risks. ${ }^{10,23}$

While radiation can treat some types of cancer pain, it does not effectively address mechanical pain, which is attributable to abnormal motion from diseased bone and increasing strain on ligamentous structures. Data from the radiation oncology literature reveal the impact of PI on outcomes. In a study comparing SFRT and MFRT, patients with SFRT and elevated SINSs ( $>11$; the threshold for PI set by the authors) were reported to have more adverse outcomes following treatment. ${ }^{16}$ In the current study, within the radiation-only cohort, 85 patients $(87 \%)$ in the PI group reported mechanical back pain at the time of treatment, suggesting that they may have benefitted from a surgical consultation to discuss elective stabilization. Of note, none of the patients in the radiation-only group required surgical intervention for catastrophic mechanical failure. Because of the heterogeneity of the patient population, narcotic use among this cohort was not examined, but future studies should investigate whether radiation reduces narcotic use specifically for mechanical back pain due to spinal metastases.

Our data are limited to a single academic medical institution and are based on a small sample size, and further studies should investigate the natural history of metastatic spine disease. Of note, preoperative KPS was not associated with an impact on survival, suggesting that the degree of preoperative disability was similar between groups. While this argues against more progressive disease in the surgical cohort, further studies may shed light on this complex population. Because of the heterogeneity of our population, prescriptive treatment regimens can vary widely between patients based on their primary oncological issue, and the timing of surgical evaluation may be related more to systemic progression of disease and nonoperative treatment failure.

One goal of this study was to examine care patterns in patients with metastatic spine disease in an attempt to identify the participation and timing of various subspecialties in this population, as well as potential areas for improvement. In total, 235 patients with spinal metastases were reviewed. One hundred nine patients had undergone surgery with or without radiation and 126 patients had received radiation alone, and all met criteria for inclusion. There was significant disparity in the timing of the involvement of the various subspecialties during the care process. Among the surgical patients, 34 patients had a history of preoperative radiation to the surgical site but ultimately required surgical stabilization. In the remaining 75 , surgical intervention preceded radiation.

Retrospective analysis of SINSs revealed that surgical consultation in the radiation-only treatment groups may 


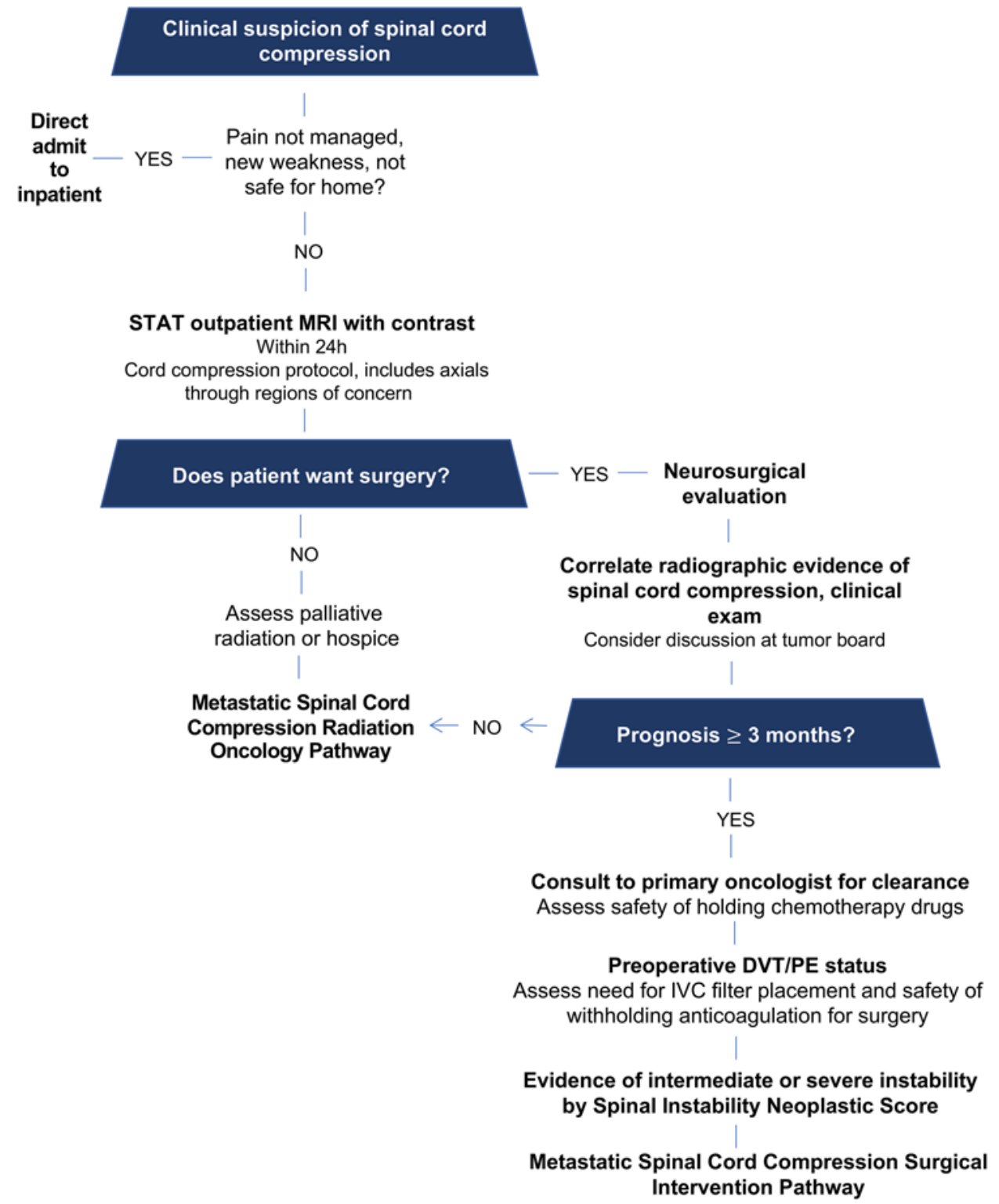

FIG. 1. Pathway for management of patients with spinal metastatic disease. The pathway is currently in use at our institution for the triage and management of patients with complex metastatic disease in the spine. The goal of the pathway is to offer guidelines for the initial management of spinal metastatic disease and to encourage early consultation of neurosurgery and radiation oncology. DVT = deep vein thrombosis; IVC = inferior vena cava; PE = pulmonary embolism. Figure is available in color online only.

be low at our institution. Among patients treated with radiation alone, 98 were found to have PI (SINS 7-12). Five patients in this cohort had neurosurgical or orthopedic surgical consultation. Among the 5 patients who had surgical consultation, no surgery was recommended.

Given the increasing complexity of the patient population and ever-changing evidence-based protocols, a multidisciplinary approach is paramount for treatment selection. In an effort to improve communication among such groups, we have instituted a metastatic epidural spinal cord compression pathway for general access (Fig. 1). This pathway highlights the key diagnostic considerations for patients with epidural cord compression and emphasizes the importance of comprehensive imaging; steroid ad- ministration; treatment of cancer-related pain; documentation of neurological, bowel, and bladder status prior to Foley catheterization; and early consultation with surgery, medical oncology, and radiation oncology. The pathway is meant for use in the emergency department, and an auxiliary pathway is provided for the outpatient system. A comprehensive spine center was recently established to reduce the time from referral to scheduling an appointment with a spine surgeon. While this system is in its early stages, it incorporates an administrative structure to prevent patients from falling through the cracks. Timely scheduling (less than 48 hours) for surgical consultation should increase referrals from radiation oncology, as providers can offer a structured plan for patients facing difficult decisions. 
Another possible point of improvement is to involve the radiologist in the early detection of patients with PI. Five of the six SINS scoring variables (location, posterior element involvement, bone quality, deformity, and extent of vertebral body involvement) are purely radiographic. A modified radiographic SINS (rSINS) can be used to increase awareness of instability for medical and radiation oncologists if included in the imaging report. We hypothesize that if an rSINS is used instead of generic language, such as "correlate with clinical exam," radiation and medical oncologists can identify patients with PI and refer them for early surgical consultation. Early detection and a scheduled clinic appointment with a spine surgeon will allow another member of the team to be involved in the decision-making process: the patient. Patients should not have to make complex decisions about metastatic disease in the middle of the night or when they are in extremis. Improved surgical consultation gives patients the chance to consider their options and decide what risks they are willing to take for their cancer care.

This retrospective study is limited to the experience of a single academic institution, and the results may not be generalizable; however, our data suggest that it may be worthwhile to examine this process. It should also be emphasized that our results do not take into account unofficial consultations (e.g., phone call or email), which are known to happen with variable frequency. In the current study, we used the SINS as a proxy for patients who may have benefited from surgical consultation. Scoring systems, while designed to standardize treatment and provide a framework for clinical decision-making, do not supplant clinical judgment, and the SINS is one of many factors involved in surgical consultation. Doubtless many of these patients would not have needed surgery. Further research is needed to document how the spine center, metastatic pathway, and other interventions impact the overall care of this population.

\section{Conclusions}

Surgery and radiation are two pillars of management for metastatic spine disease, and while scoring systems have been used to clarify treatment strategies, decision-making remains highly complex. In this study, we attempted to examine treatment and collaboration patterns for patients with metastatic spine disease at our institution. The results of this study suggest that surgical consultation may be underutilized at our institution, and we propose several strategies for improvement in communication between the multiple disciplines often required in the care of this complicated patient group. While the data represent the experience of a single institution, we believe they urge other institutions to think introspectively about this process, which indeed involves highly complex decision-making.

\section{References}

1. Arguello F, Baggs RB, Duerst RE, Johnstone L, McQueen K, Frantz CN: Pathogenesis of vertebral metastasis and epidural spinal cord compression. Cancer 65:98-106, 1990

2. Bilsky M, Fischer CG, Gokasalan ZL, Fehlings MG, Boriani S, Polly D, et al: The Spinal Instability Neoplastic Score (SINS): an analysis of reliability and validity from the Spine
Oncology Study Group. Int J Radiat Oncol Biol Phys 78:S263, 2010

3. Chan NK, Abdullah KG, Lubelski D, Steinmetz MP, Benzel EC, Shin JH, et al: Stereotactic radiosurgery for metastatic spine tumors. J Neurosurg Sci 58:37-44, 2014

4. Choi D, Bilsky M, Fehlings M, Fisher C, Gokaslan Z: Spine oncology-metastatic spine tumors. Neurosurgery 80 (3S):S131-S137, 2017

5. Fisher CG, DiPaola CP, Ryken TC, Bilsky MH, Shaffrey CI, Berven SH, et al: A novel classification system for spinal instability in neoplastic disease: an evidence-based approach and expert consensus from the Spine Oncology Study Group. Spine (Phila Pa 1976) 35:E1221-E1229, 2010

6. Fisher CG, Versteeg AL, Schouten R, Boriani S, Varga PP, Rhines LD, et al: Reliability of the spinal instability neoplastic scale among radiologists: an assessment of instability secondary to spinal metastases. AJR Am J Roentgenol 203:869-874, 2014

7. Fourney DR, Gokaslan ZL: Anterior approaches for thoracolumbar metastatic spine tumors. Neurosurg Clin N Am 15:443-451, 2004

8. Fox S, Spiess M, Hnenny L, Fourney DR: Spinal Instability Neoplastic Score (SINS): reliability among spine fellows and resident physicians in orthopedic surgery and neurosurgery. Global Spine J 7:744-748, 2017

9. Gerszten PC, Burton SA, Ozhasoglu C, Welch WC: Radiosurgery for spinal metastases: clinical experience in 500 cases from a single institution. Spine (Phila Pa 1976) 32:193-199, 2007

10. Ghogawala Z, Mansfield FL, Borges LF: Spinal radiation before surgical decompression adversely affects outcomes of surgery for symptomatic metastatic spinal cord compression. Spine (Phila Pa 1976) 26:818-824, 2001

11. Gokaslan ZL, York JE, Walsh GL, McCutcheon IE, Lang FF, Putnam JB Jr, et al: Transthoracic vertebrectomy for metastatic spinal tumors. J Neurosurg 89:599-609, 1998

12. Hoskin P, Misra V, Hopkins K, Holt T, Brown G, Arnott S, et al: SCORAD III: randomized noninferiority phase III trial of single-dose radiotherapy (RT) compared to multifraction $\mathrm{RT}$ in patients (pts) with metastatic spinal canal compression (SCC). J Clin Oncol 35:LBA10004, 2017

13. Howell DD, James JL, Hartsell WF, Suntharalingam M, Machtay M, Suh JH, et al: Single-fraction radiotherapy versus multifraction radiotherapy for palliation of painful vertebral bone metastases-equivalent efficacy, less toxicity, more convenient: a subset analysis of Radiation Therapy Oncology Group trial 97-14. Cancer 119:888-896, 2013

14. Hsu W, Sciubba DM, Sasson AD, Khavkin Y, Wolinsky JP, Gailloud P, et al: Intraoperative localization of thoracic spine level with preoperative percutaneous placement of intravertebral polymethylmethacrylate. J Spinal Disord Tech 21:72-75, 2008

15. Klimo P Jr, Schmidt MH: Surgical management of spinal metastases. Oncologist 9:188-196, 2004

16. Lam TC, Uno H, Krishnan M, Lutz S, Groff M, Cheney M, et al: Adverse outcomes after palliative radiation therapy for uncomplicated spine metastases: role of spinal instability and single-fraction radiation therapy. Int J Radiat Oncol Biol Phys 93:373-381, 2015

17. Laufer I, Rubin DG, Lis E, Cox BW, Stubblefield MD, Yamada Y, et al: The NOMS framework: approach to the treatment of spinal metastatic tumors. Oncologist 18:744-751, 2013

18. Lee CS, Jung CH: Metastatic spinal tumor. Asian Spine J 6:71-87, 2012

19. Moussazadeh N, Laufer I, Yamada Y, Bilsky MH: Separation surgery for spinal metastases: effect of spinal radiosurgery on surgical treatment goals. Cancer Contr 21:168-174, 2014

20. Padalkar P, Tow B: Predictors of survival in surgically treat- 
ed patients of spinal metastasis. Indian J Orthop 45:307313, 2011

21. Patchell RA, Tibbs PA, Regine WF, Payne R, Saris S, Kryscio RJ, et al: Direct decompressive surgical resection in the treatment of spinal cord compression caused by metastatic cancer: a randomised trial. Lancet 366:643-648, 2005

22. Versteeg AL, van der Velden JM, Verkooijen HM, van Vulpen M, Oner FC, Fisher CG, et al: The effect of introducing the Spinal Instability Neoplastic Score in routine clinical practice for patients with spinal metastases. Oncologist 21:95-101, 2016

23. Wise JJ, Fischgrund JS, Herkowitz HN, Montgomery D, Kurz LT: Complication, survival rates, and risk factors of surgery for metastatic disease of the spine. Spine (Phila Pa 1976) 24:1943-1951, 1999

\section{Disclosures}

Dr. Saifi has direct stock ownership in Vertera/NuVasive. Dr. Ozturk is a consultant for DePuy Synthes.

\section{Author Contributions}

Conception and design: Sullivan, Schuster. Acquisition of data: Sullivan, Ramayya, McShane, Chen, Schuster, Jones. Analysis and interpretation of data: Sullivan, Albayar, Ramayya, Jones, Ozturk. Drafting the article: Sullivan, Albayar, Jones, Ozturk. Critically revising the article: Albayar, Marcotte, Malhotra, Ali, Chen, Grady. Reviewed submitted version of manuscript: Marcotte, Janjua, Saifi, Schuster.

\section{Supplemental Information}

\section{Previous Presentations}

Portions of this work were presented as an oral abstract at the Pan-Philadelphia Neurosurgery Conference held in Philadelphia, PA, on December 1, 2017.

\section{Correspondence}

Patricia Zadnik Sullivan: University of Pennsylvania, Philadelphia,PA.patricia.zadnik@pennmedicine.upenn.edu. 